\title{
Non-minimal coupling as a mechanism for spontaneous symmetry breaking on the brane
}

\author{
Orfeu Bertolami ${ }^{\star a}$ and Carla Carvalho ${ }^{a b \ddagger}$ \\ ${ }^{a}$ Departamento de Física, Instituto Superior Técnico \\ Avenida Rovisco Pais 1, 1049-001 Lisboa, Portugal \\ ${ }^{b}$ Laboratoire Physique Théorique, \\ Bâtiment 210, 91405 Orsay, France \\ E-mail: orfeudcosmos.ist.utl.pt, ccarvalhodist.edu
}

\begin{abstract}
Motivated by the dimensional asymmetry characteristic of braneworlds, we populate the bulk spacetime with matter scalar fields, both real and complex, and couple them non-minimally to gravity. We derive the effective equations of motion on the brane and realize the case when the fields acquire a non-vanishing vacuum expectation value. This entails a change in the effective cosmological constant and in the effective mass of the scalar fields. We find that the non-minimal coupling provides a mechanism for generating spontaneous symmetry breaking at Planck energies on the brane. This mechanism is however rendered unviable for the generation of masses at the energy scales of the standard model.
\end{abstract}

From Quantum to Emergent Gravity: Theory and Phenomenology June 11-15 2007

Trieste, Italy

\footnotetext{
*Speaker.

${ }^{\dagger}$ Also at Centro de Física dos Plasmas, Instituto Superior Técnico.

$¥$ Also at Centro de Física dos Plasmas, Instituto Superior Técnico.
} 


\section{Introduction}

Braneworld cosmological models, by realizing the four-dimensional universe entirely in a hypersurface of a higher dimensional space, are intrinsically dimensionally asymmetric. The emergence of the difference between the parallel and the orthogonal directions to this hypersurface seems to reverberate the Goldstone mechanism, where massless multiplets are imparted with a mass in the direction along which symmetry is spontaneously broken. Here we propose to use spontaneous symmetry breaking (SSB) of bulk scalar fields to relate the mass measured on the brane to a bulk mechanism and thus look for signatures of extra dimensions. This is the opposite idea to the Dvali-Shifman mechanism, where a non-confinement Higgs (spontaneously broken symmetry) phase is found on the brane, whereas a confinement (restored symmetry) phase is preserved in the bulk [1]. Moreover, we couple the bulk scalar fields non-minimally to the Ricci scalar. This is the simplest interaction which yields a canonical kinetic term. Furthermore, it reduces to Brans-Dicke up to a field transformation for a vanishing vacuum expectation value (vev). Recently, a variation of the Dvali-Shifman mechanism triggered by the geometry of five-dimensional anti-de Sitter was applied to the study of the dynamics along the direction normal to the brane of a scalar field non-minimally coupled to gravity [2].

This contribution reports on a recent study where we consider both real and complex scalar fields in the bulk space and examine their implication in the mechanism of SSB on the brane upon acquiring a non-vanishing bulk vev [3]. Furthermore, when studying the case of the complex scalar field, we also consider a minimally coupled $U(1)$ gauge field so that spontaneous breaking of the gauge symmetry can take place. After establishing how bulk quantities induce quantities on the brane, we compute the equations of motion induced on the brane starting from a bulk action. The resulting Einstein equations provide the relations between the induced geometry on the brane and the matter fields therein. Furthermore, we observe that matter a priori localized on the brane, such as the brane tension, will only interact gravitationally with the bulk matter fields induced on the brane when a non-minimal coupling exists. Otherwise, brane and bulk matter fields do not see each other. The non-minimal coupling also reinforces the canonical mechanism of spontaneous symmetry breaking at very high energies via the interaction of the brane tension with the scalar fields. Moreover, we discuss whether the SSB mechanism could suffice to generate masses on the brane and thus provide a localization mechanism. The four-dimensional masses, both of the scalar and the gauge fields, induced on the brane are found to be of order the four-dimensional Planck mass. Taking the inverse of the mass as a measure of the confinement to the brane [4], it follows that the range of the induced interaction is short about the brane, which suggests the localization of the bulk fields about the position of the brane.

In this contribution we consider a real scalar field in the bulk and non-minimally coupled to gravity via the Ricci scalar. [For the case of the complex scalar field, we refer the reader to Ref. [3].] Following the procedure described in the Appendix, we derive the induced equations on the brane from the bulk action. In particular, we derive the effective potential on the brane and compute the effective mass of the scalar field induced on the brane upon taking a non-vanishing vev in the bulk. Finally, we analyse the implications of our results for a mechanism of localization of matter on the brane. We keep the number of space dimensions $d$ arbitrary, rendering the results valid for any codimension-one brane. 


\section{Real Scalar Field Non-minimally Coupled in the Bulk}

In this section we study the case of a bulk, real scalar field $\phi$. In addition to the EinsteinHilbert term and the canonical kinetic and potential terms of $\phi$, we consider an interaction term with a non-minimal coupling of $\phi$ to the Ricci scalar

$$
\mathscr{L}=\frac{1}{\kappa_{(5)}^{2}} R-2 \Lambda+\xi \phi^{2} R-\frac{1}{2} g^{\mu v}\left(\nabla_{\mu} \phi\right)\left(\nabla_{v} \phi\right)-V\left(\phi^{2}\right) .
$$

Here, $\kappa_{(5)}^{2}=8 \pi G_{N(5)}=1 / M_{P l}^{3}$ is the five-dimensional gravitational coupling constant and $\xi$ is a dimensionless coupling constant which measures the non-minimal interaction. In the cosmological constant term $\Lambda=\Lambda_{(5)}+\Lambda_{(4)}$ we have included both the bulk vacuum value $\Lambda_{(5)}$ and that of the brane $\Lambda_{(4)}$, described by a brane tension $\sigma$ localized at the position of the brane, $\Lambda_{(4)}=\sigma \delta(N)$.

\subsection{The induced dynamics on the brane}

First we derive the equations of motion for both the scalar field and the graviton as measured by an observer localized on and confined to the brane. This procedure follows closely the techniques developed in Ref. [5] for a vector field and in particular uses the results derived therein, which for completion are included in an appendix.

By varying the action with respect to the metric, we obtain the Einstein equation in the bulk

$$
\left(\frac{1}{\kappa_{(5)}^{2}}+\xi \phi^{2}\right) G_{\mu v}+\Lambda g_{\mu v}=\frac{1}{2} T_{\mu \nu}^{(\phi)}+\xi \Sigma_{\mu \nu}^{(\phi)},
$$

where

$$
T_{\mu \nu}^{(\phi)}=\left(\nabla_{\mu} \phi\right)\left(\nabla_{\nu} \phi\right)+g_{\mu v}\left[-\frac{1}{2} g^{\alpha \beta}\left(\nabla_{\alpha} \phi\right)\left(\nabla_{\beta} \phi\right)-V\left(\phi^{2}\right)\right]
$$

is the stress-energy tensor associated with $\phi$ and

$$
\Sigma_{\mu \nu}^{(\phi)}=\nabla_{\mu} \nabla_{\nu} \phi^{2}-g_{\mu \nu} g^{\alpha \beta} \nabla_{\alpha} \nabla_{\beta} \phi^{2}
$$

is the contribution from the interaction term. We note the $\phi$-dependence of the five-dimensional gravitational coupling constant akin to that of the Brans-Dicke formulation. For the equation of motion for the $\phi$ field, obtained by varying the action with respect to $\phi$, we find that

$$
g^{\mu v} \nabla_{\mu} \nabla_{v} \phi-\frac{\partial V}{\partial \phi}+2 \xi \phi R=0 .
$$

We now proceed to project the equations in the directions parallel (denoted by $A$ ) and orthogonal (denoted by $N$ ) to the surface of the brane, finding for the stress-energy tensor $\mathbf{T}^{(\phi)}$ that

$$
\begin{aligned}
T_{A B}^{(\phi)} & =\left(\nabla_{A} \phi\right)\left(\nabla_{B} \phi\right)+g_{A B}\left[-\frac{1}{2}\left[\left(\nabla_{C} \phi\right)^{2}+\left(\nabla_{N} \phi\right)^{2}\right]-V\left(\phi^{2}\right)\right], \\
T_{A N}^{(\phi)} & =\left(\nabla_{A} \phi\right)\left(\nabla_{N} \phi\right), \\
T_{N N}^{(\phi)} & =\left(\nabla_{N} \phi\right)\left(\nabla_{N} \phi\right)+g_{N N}\left[-\frac{1}{2}\left[\left(\nabla_{C} \phi\right)^{2}+\left(\nabla_{N} \phi\right)^{2}\right]-V\left(\phi^{2}\right)\right],
\end{aligned}
$$


and similarly for the source tensor $\Sigma_{\mu \nu}^{(\phi)}$ that

$$
\begin{aligned}
& \Sigma_{A B}^{(\phi)}=\left(\nabla_{A} \nabla_{B}+K_{A B} \nabla_{N}\right) \phi^{2}-g_{A B}\left(\nabla_{C}^{2}+\nabla_{N}^{2}+K \nabla_{N}\right) \phi^{2}, \\
& \Sigma_{A N}^{(\phi)}=\left(\nabla_{A} \nabla_{N}-K_{A}^{B} \nabla_{B}\right) \phi^{2} \\
& \Sigma_{N N}^{(\phi)}=\nabla_{N} \nabla_{N} \phi^{2}-g_{N N}\left(\nabla_{C}^{2}+\nabla_{N}^{2}+K \nabla_{N}\right) \phi^{2} .
\end{aligned}
$$

Equating the $(A B)$ components of the decomposition of the Einstein tensor and of the source terms from the scalar field $\phi$, we find for the Einstein equation parallel projected on to the brane that

$$
\begin{aligned}
& \left(\frac{1}{\kappa_{(5)}^{2}}+\xi \phi^{2}\right)\left[G_{A B}^{(i n d)}+2 K_{A C} K_{B}^{C}-K_{A B} K-K_{A B, N}-\frac{1}{2} g_{A B}\left(-K_{C D} K^{C D}-K^{2}-2 K_{, N}\right)\right] \\
= & \frac{1}{2}\left(\nabla_{A} \phi\right)\left(\nabla_{B} \phi\right)+\frac{1}{2} g_{A B}\left[-\frac{1}{2}\left[\left(\nabla_{C} \phi\right)^{2}+\left(\nabla_{N} \phi\right)^{2}\right]-V\left(\phi^{2}\right)\right]-g_{A B} \Lambda \\
+ & \xi\left[\left(\nabla_{A} \nabla_{B}+K_{A B} \nabla_{N}\right) \phi^{2}-g_{A B}\left(\nabla_{C}^{2}+\nabla_{N}^{2}+K \nabla_{N}\right) \phi^{2}\right] .
\end{aligned}
$$

To obtain the matching condition for the extrinsic curvature across the brane, we integrate the $(A B)$ component of the Einstein equation in the coordinate normal to the brane. For a $Z_{2}$-symmetric brane, we obtain for the $(A B)$ matching condition across the brane

$$
\begin{aligned}
& \int_{-\delta}^{+\delta} d N\left(\frac{1}{\kappa_{(5)}^{2}}+\xi \phi^{2}\right)\left(-K_{A B, N}+g_{A B} K_{, N}\right) \\
= & \int_{-\delta}^{+\delta} d N\left[-g_{A B} \Lambda_{(4)}+\xi\left(\left(K_{A B}-g_{A B} K\right) \nabla_{N} \phi^{2}-g_{A B} \nabla_{N}^{2} \phi^{2}\right)\right],
\end{aligned}
$$

which yields

$$
\left(\frac{1}{\kappa_{(5)}^{2}}+\xi \phi^{2}\right)\left(-K_{A B}+g_{A B} K\right)=g_{A B}\left(-\frac{\sigma}{2}-\xi \nabla_{N} \phi^{2}\right)
$$

for $\phi^{2}$ even about the position of the brane. These provide boundary conditions for ten of the fifteen degrees of freedom. Five additional boundary conditions are provided by the matching conditions from the $(A N)$ and $(N N)$ components of the projected Einstein equations. From inspection of the $(A N)$ components we note that on the brane

$$
G_{A N}=K_{A}{ }^{B} ; B-K_{; A}=-\nabla_{B}\left(\int d N G_{A}{ }^{B}\right)=-\nabla_{B} \mathscr{T}_{A}^{B(\phi)}=0,
$$

which must vanish in order to preserve conservation of the induced stress-energy $\mathscr{T}_{A B}^{(\phi)}$ on the brane, as read off of the right-hand side of Eq. (2.10). This condition constrains four degrees of freedom. The $(N N)$ component of the Einstein equation

$$
\begin{aligned}
& \left(\frac{1}{\kappa_{(5)}^{2}}+\xi \phi^{2}\right)\left[-R^{(i n d)}-K_{C D} K^{C D}+K^{2}\right]+\Lambda_{(5)} \\
= & \frac{1}{2}\left[\frac{1}{2}\left(\nabla_{N} \phi\right)\left(\nabla_{N} \phi\right)-\frac{1}{2}\left(\nabla_{C} \phi\right)\left(\nabla_{C} \phi\right)-V\left(\phi^{2}\right)\right]-\xi\left(\nabla_{C}^{2}+K \nabla_{N}\right) \phi^{2}
\end{aligned}
$$

consists of the remaining constraint. 
Similarly, we expand the equation of motion for the scalar field $\phi$

$$
\begin{gathered}
{\left[g^{A B}\left(\nabla_{A} \nabla_{B}+K_{A B} \nabla_{N}\right)+\nabla_{N} \nabla_{N}\right] \phi-\frac{\partial V}{\partial \phi}} \\
+2 \xi \phi\left(R^{(i n d)}-K_{A B} K^{A B}-K^{2}-2 K_{, N}\right)=0 .
\end{gathered}
$$

To obtain the matching condition for $\phi$ across the brane, we integrate in the $N$ coordinate discarding all derivatives other than along $N$

$$
\int_{-\delta}^{+\delta} d N\left[K \nabla_{N} \phi+\nabla_{N} \nabla_{N} \phi-4 \xi \phi K_{, N}\right]=0 .
$$

If we assume $Z_{2}$-symmetry across the brane, the matching condition for $\phi$ becomes

$$
\nabla_{N} \phi-4 \xi K \phi=0 \text {. }
$$

Substituting Eq. (2.15) back into Eq. (2.13), we obtain for the propagation of $\phi$ on the brane that

$$
g^{A B} \nabla_{A} \nabla_{B} \phi-\frac{\partial V}{\partial \phi}+2 \xi \phi\left[R^{(i n d)}-K_{A B} K^{A B}+(1+8 \xi) K^{2}\right]=0 .
$$

Moreover, equating the matching conditions for the extrinsic curvature, Eq. (2.10), and for the scalar field $\phi$, Eq. (2.15), we can solve for $K_{A B}$ and $\nabla_{N} \phi$ to find that

$$
\begin{aligned}
K_{A B} & =-\left.\frac{1}{2} g_{A B} \sigma \frac{1 /(d-1)}{1 / \kappa_{(5)}^{2}+\xi \phi^{2}[1+8 \xi d /(d-1)]}\right|_{N=0}, \\
\left.\nabla_{N} \phi\right|_{N=0} & =-\left.2 \xi \phi \sigma \frac{d /(d-1)}{1 / \kappa_{(5)}^{2}+\xi \phi^{2}[1+8 \xi d /(d-1)]}\right|_{N=0} .
\end{aligned}
$$

We substitute Eq. (2.15) for $\nabla_{N} \phi$ and Eq. (2.16) for $\nabla_{C}^{2} \phi$ in the $(N N)$ component of the Einstein equation, Eq. (2.12), to find for $R^{(i n d)}$ that

$$
\begin{gathered}
\left(\frac{1}{\kappa_{(5)}^{2}}+\xi \phi^{2}(1+4 \xi)\right) R^{(i n d)}=\left(\frac{1}{4}+2 \xi\right)\left(\nabla_{C} \phi\right)^{2}+\frac{1}{2} V+2 \xi \phi \frac{\partial V}{\partial \phi}+\Lambda_{(5)} \\
-K_{C D} K^{C D}\left(\frac{1}{\kappa_{(5)}^{2}}+\xi \phi^{2}(1-4 \xi)\right)+K^{2}\left(\frac{1}{\kappa_{(5)}^{2}}+\xi \phi^{2}\left(1-32 \xi^{2}\right)\right) .
\end{gathered}
$$

Similarly, from Eq. (2.8) we find for the Einstein equation induced on the brane that

$$
\begin{aligned}
G_{A B}^{(i n d)} & =\left(\frac{1}{\kappa_{(5)}^{2}}+\xi \phi^{2}\right)^{-1}\left[\left(\frac{1}{2}+2 \xi\right)\left(\nabla_{A} \phi\right)\left(\nabla_{B} \phi\right)+2 \xi \phi \nabla_{A} \nabla_{B} \phi\right] \\
& -g_{A B}\left[R^{(i n d)}+K^{2} \frac{-d^{2}+d+4}{2 d^{2}}\right],
\end{aligned}
$$

with $R^{(i n d)}$ given by Eq. (2.19).

Using the equations derived above, we realize the case where the scalar field acquires a nonvanishing vev $\langle\phi\rangle$ which minimizes the effective potential. This can induce spontaneous symmetry breaking when the scalar field is coupled to a gauge field, thus endowing the latter with a mass, 
as discussed in Ref. [3]. Here, however, a non-vanishing vev will entail a change in the effective cosmological constant and in the effective mass of the scalar field. Moreover, once the scalar field acquires a vev, no direction on the brane can be selected, which implies that $\nabla_{A}\langle\phi\rangle=0$. Consequently, Lorentz symmetry breaking cannot take place in the presence of a bulk scalar field only. [See Ref. [5] for the case of an explicit violation of Lorentz symmetry due to a non-vanishing vev for a vector field.]

We can read off of the induced Einstein equation the effective cosmological constant, which would comprise all the terms proportional to the induced metric which do not vanish when all the contributions from the matter fields vanish. However, in the case that the matter fields acquire a non-vanishing vev, the effective cosmological constant will contain the contribution of the matter fields at the corresponding non-vanishing value. It follows that

$$
\begin{gathered}
\Lambda_{e f f}\left(\frac{1}{\kappa_{(5)}^{2}}+\xi\langle\phi\rangle^{2}(1+4 \xi)\right)=\frac{1}{2} V\left(\langle\phi\rangle^{2}\right)+\left.2 \xi\langle\phi\rangle \frac{\partial V}{\partial \phi}\right|_{\phi=\langle\phi\rangle}+\Lambda_{(5)} \\
+\left.K^{2}\right|_{\phi=\langle\phi\rangle}\left[\left(\frac{1}{\kappa_{(5)}^{2}}+\xi\langle\phi\rangle^{2}\right) \frac{d^{2}-d+4}{2 d^{2}}+(2 \xi\langle\phi\rangle)^{2}\left(\frac{-d^{2}+3 d+4}{2 d^{2}}-8 \xi\right)\right] .
\end{gathered}
$$

We thus observe that a non-vanishing vev in the bulk generates in the gravitational sector a contribution to the cosmological constant on the brane.

\subsection{The effective potential on the brane}

Whether a non-vanishing vev for the scalar field can be observed on the brane depends on the form of the effective potential $V_{\text {eff }}\left(\phi^{2}\right)$. The parameters of the potential will influence the magnitude of its minimum and consequently the mass of the scalar field $\phi$ measured on the brane, defined as the value of the second derivative of the effective potential evaluated at the vev of the scalar field, $\langle\phi\rangle$. We first determine the effective potential measured on the brane and then proceed to study the conditions for a non-vanishing vev.

The evolution of $\phi$ on the brane, as described by Eq. (2.16) and with $R^{(i n d)}$ given by Eq. (2.19),

$$
g^{A B} \nabla_{A} \nabla_{B} \phi-\frac{\partial V_{e f f}}{\partial \phi}+\left(\frac{1}{4}+2 \xi\right)\left(\nabla_{C} \phi\right)^{2} \frac{2 \xi \phi}{1 / \kappa_{(5)}^{2}+\xi \phi^{2}(1+4 \xi)}=0
$$

is determined by a damping term as well as by the effective potential induced on the brane. Here,

$$
\begin{aligned}
-\frac{\partial V_{e f f}}{\partial \phi} & =\frac{1}{1 / \kappa_{(5)}^{2}+\xi \phi^{2}(1+4 \xi)}\left\{-\frac{\partial V}{\partial \phi}\left(\frac{1}{\kappa_{(5)}^{2}}+\xi \phi^{2}\right)\right. \\
& +2 \xi \phi\left[\frac{1}{2} V+\Lambda_{(5)}\right] \\
& \left.+2 \xi \phi K^{2}\left[\frac{1}{\kappa_{(5)}^{2}}\left(-\frac{2}{d}+2+8 \xi\right)+\xi \phi^{2}\left(-\frac{2}{d}+2+12 \xi\right)\right]\right\}
\end{aligned}
$$

where $V\left(\phi^{2}\right)$ is the bulk potential, which is assumed to have a Higgs type form $V(\phi)=\mu_{(5)}^{2}\left(\phi^{2} / 2\right)+$ $\lambda_{(5)}\left(\phi^{4} / 4\right)$ with $\lambda_{(5)}>0$. We compute $V_{e f f}$ by integrating Eq. (2.23) to find that

$$
V_{e f f}\left(\phi^{2}\right)=\phi^{2}\left[\frac{\mu_{(5)}^{2}}{2} \frac{1}{1+4 \xi}-\frac{\mu_{(5)}^{2}}{4} \frac{1}{1+4 \xi}+\lambda_{(5)} \frac{1}{\xi \kappa_{(5)}^{2}} \frac{1}{2(1+4 \xi)^{2}}\left(\frac{1}{4}+4 \xi\right)\right]
$$




$$
\begin{aligned}
+ & \phi^{4} \frac{\lambda(5)}{4}\left[\frac{1}{1+4 \xi}-\frac{1}{4(1+4 \xi)}\right] \\
+ & \ln \left[1+\xi \kappa_{(5)}^{2} \phi^{2}(1+4 \xi)\right] \times \\
& \times\left[\mu_{(5)}^{2} \frac{1}{\xi \kappa_{(5)}^{2}} \frac{1}{2(1+4 \xi)^{2}}\left(\frac{1}{2}+4 \xi\right)-\lambda_{(5)} \frac{1}{\left(\xi \kappa_{(5)}^{2}\right)^{2}} \frac{1}{2(1+4 \xi)^{3}}\left(\frac{1}{4}+4 \xi\right)\right. \\
& \left.-\frac{1}{1+4 \xi} \Lambda_{(5)}\right] \\
+ & \frac{\sigma^{2}}{16} \frac{d^{2}}{(d+1)(d-1)} \times \\
& \times\left[\frac{[-2 / d+2(1+4 \xi)](8 d /(d-1))-4}{1+8 \xi d /(d-1)} \frac{1}{1 / \kappa_{(5)}^{2}+\xi \phi^{2}[1+8 \xi d /(d-1)]}\right. \\
& \left.-\frac{\kappa_{(5)}^{2}}{\xi} \frac{d-1}{d+1}\left(-\frac{2}{d}+1+8 \xi\right) \ln \left[\frac{1+\xi \kappa_{(5)}^{2} \phi^{2}(1+4 \xi)}{1+\xi \kappa_{(5)}^{2} \phi^{2}[1+8 \xi d /(d-1)]}\right]\right]
\end{aligned}
$$

for $\xi \neq-1 / 4$. Notice that, in the limit when $\xi \rightarrow 0$, one recovers the original bulk potential with an extra term on the brane tension, $V\left(\phi^{2}\right)+(3 / 4) \sigma^{2} \kappa_{(5)}^{2} d^{2} /[(d+1)(d-1)]$. It is natural to expect that there exists a hierarchy of scales depending on whether the vev $\langle\phi\rangle$ is related to the Standard Model (SM) scale or the grand unified theory scale. Thus, for $\left|\xi \phi^{2}\right| \ll 1 / \kappa_{(5)}^{2}$, we can expand the denominator of the first term in $\sigma$ about $1 / \kappa_{(5)}^{2}$, keeping terms up to order six in $\phi,\left(\xi \phi^{2}\right)^{3}$. The effective potential can thus be written as

$$
\begin{aligned}
V_{e f f}\left(\phi^{2}\right)= & \phi^{2}\left\{\frac{\mu_{(5)}^{2}}{2} \frac{1}{1+4 \xi}-\frac{\mu_{(5)}^{2}}{4} \frac{1}{1+4 \xi}+\lambda(5) \frac{1}{\xi \kappa_{(5)}^{2}} \frac{1}{2(1+4 \xi)}\left(\frac{1}{4}+4 \xi\right)\right. \\
& \left.-\frac{1}{16} \sigma^{2} \kappa_{(5)}^{2}\left(\xi \kappa_{(5)}^{2}\right) \frac{d^{2}}{(d+1)(d-1)}\left[\left(-\frac{2}{d}+2(1+4 \xi)\right) \frac{8 d}{d-1}-4\right]\right\} \\
+ & \phi^{4}\left\{\frac{\lambda(5)}{4} \frac{1}{1+4 \xi}-\frac{\lambda(5)}{4} \frac{1}{4(1+4 \xi)}\right. \\
& \left.+\frac{1}{16} \sigma^{2} \kappa_{(5)}^{2} \frac{1}{2}\left(\xi \kappa_{(5)}^{2}\right)^{2} \frac{d^{2}}{(d+1)(d-1)}\left[\left(-\frac{2}{d}+2(1+4 \xi)\right) \frac{8 d}{d-1}-4\right]\left(1+\xi \frac{8 d}{d-1}\right)\right\} \\
- & O\left[\phi^{6}\right] \frac{1}{16} \sigma^{2} \kappa_{(5)}^{2} \frac{1}{6}\left(\xi \kappa_{(5)}^{2}\right)^{3} \\
+ & \ln \left[1+\xi \kappa_{(5)}^{2} \phi^{2}(1+4 \xi)\right] \times \\
& \times\left[\mu_{(5)}^{2} \frac{1}{\xi \kappa_{(5)}^{2}} \frac{1}{2(1+4 \xi)^{2}}\left(\frac{1}{2}+4 \xi\right)-\lambda \lambda_{(5)} \frac{1}{\left(\xi \kappa_{(5)}^{2}\right)^{2}} \frac{1}{2(1+4 \xi)^{3}}\left(\frac{1}{4}+4 \xi\right)\right. \\
& \left.-\frac{1}{1+4 \xi} \Lambda_{(5)}\right] \\
+ & \frac{1}{16} \sigma^{2} \kappa_{(5)}^{2} \frac{d^{2}}{(d+1)(d-1)} \times \\
& \times\left[\frac{[-2 / d+2(1+4 \xi)](8 d /(d-1))-4}{1+8 \xi d /(d-1)}\right.
\end{aligned}
$$




$$
\left.-\frac{1}{\xi} \frac{d-1}{d+1}\left(-\frac{2}{d}+1+8 \xi\right) \ln \left[\frac{1+\xi \kappa_{(5)}^{2} \phi^{2}(1+4 \xi)}{1+\xi \kappa_{(5)}^{2} \phi^{2}[1+8 \xi d /(d-1)]}\right]\right] .
$$

We thus observe that, up to sub-dominant logarithmic terms, the effective potential is of the form $V_{e f f}=\mu_{e f f}^{2}\left(\phi^{2} / 2\right)+\lambda_{e f f}\left(\phi^{4} / 4\right)+O\left[\phi^{6}\right]$, where

$$
\begin{aligned}
& \mu_{e f f}^{2} \sim \mu_{(5)}^{2}+\lambda_{(5)} \frac{1}{\xi \kappa_{(5)}^{2}}-\sigma^{2} \xi \kappa_{(5)}^{4}, \\
& \lambda_{\text {eff }} \sim \lambda_{(5)}+\sigma^{2} \xi^{2} \kappa_{(5)}^{6} .
\end{aligned}
$$

If $\mu_{e f f}^{2}<0$ and $\lambda_{\text {eff }}>0$, then one expects a non-vanishing vev for the scalar field. The first condition guarantees that a non-vanishing minimum exists, whereas the second condition guarantees that such minimum is finite. Conversely, if $\mu_{e f f}^{2}>0$ and $\lambda_{e f f}>0$, then symmetry is always unbroken. Thus, imposing that $\lambda_{\text {eff }}>0$, it follows that $\lambda_{(5)}>-\sigma^{2} \xi^{2} \kappa_{(5)}^{6}$. Consequently, in order to verify the condition $\mu_{e f f}^{2}<0$, we must have that $\mu_{(5)}^{2}<-2 \sigma^{2} \xi \kappa_{(5)}^{4}$.

We notice that the bulk scalar field $\phi$, being a five-dimensional field, has dimension $[\phi]=M^{3 / 2}$. Accordingly, $\mu_{(5)}$ has dimension of mass and $\lambda_{(5)}$ dimensions of inverse of mass. In order to recover characteristically four-dimensional quantities, we define the four-dimensional scalar field $\Phi$ as the rescaling of $\phi$ by an appropriate mass scale $M_{\phi}$. In the mode expansion of a bulk field, this mass can be identified with the mode function dependent on the direction $N$ evaluated at the position of the brane in the bulk. Thus, for $\phi=M_{\phi}^{1 / 2} \Phi$, the induced equation of motion for $\Phi$ on the brane becomes

$$
g^{A B} \nabla_{A} \nabla_{B} \Phi-\frac{1}{M_{\phi}} \frac{\partial V_{e f f}}{\partial \Phi}+\left(\frac{1}{4}+2 \xi\right)\left(\nabla_{C} \Phi\right)^{2} \frac{2 \xi M_{\phi} \Phi}{1 / \kappa_{(5)}^{2}+\xi M_{\phi} \Phi^{2}(1+4 \xi)}=0
$$

Consequently, the parameters of the effective potential will scale as

$$
\frac{1}{M_{\phi}} V_{e f f}\left(\Phi^{2}\right)=\mu_{e f f}^{2} \frac{\Phi^{2}}{2}+\lambda_{e f f} M_{\phi} \frac{\Phi^{4}}{4}+M_{\phi}^{2} O\left[\Phi^{6}\right]
$$

with equations (2.26) and (2.27) becoming

$$
\begin{aligned}
\mu_{e f f}^{2} & \sim \mu^{2}-2 \sigma^{2} \xi \kappa_{(5)}^{4}, \\
M_{\phi} \lambda_{e f f} & \sim \lambda+M_{\phi} \sigma^{2} \xi^{2} \kappa_{(5)}^{6},
\end{aligned}
$$

where $\mu=\mu_{(5)}$ and $\lambda=M_{\phi} \lambda_{(5)}$. Here, for $\xi>0$ we have two possible mechanisms for the generation of a non-vanishing vev: the canonical way, via the potential associated with the scalar field, and the braneworld way, via the interaction of the scalar field with the brane tension. For the latter to be viable in the context of the SM, then

$$
\left|\frac{\mu_{e f f}^{2}}{M_{\phi} \lambda_{e f f}}\right| \sim \frac{1}{\xi M_{\phi}} \frac{1}{\kappa_{(5)}^{2}}
$$

must be of order $\mathrm{TeV}^{2}$, and $\langle\Phi\rangle=246 \mathrm{GeV}$. However, in order to recover the four-dimensional gravitational coupling constant in Eq. (2.20), we find from the $\phi$ contribution that $M_{P l(4)}^{-2}=\kappa_{(5)}^{2} M_{\phi}$, and hence that

$$
M_{P l}^{3} \equiv \frac{1}{\kappa_{(5)}^{2}}=M_{P l(4)}^{2} M_{\phi} .
$$


This implies that

$$
\left|\frac{\mu_{e f f}^{2}}{M_{\phi} \lambda_{e f f}}\right| \sim \frac{1}{\xi} M_{P l(4)}^{2} \gg T e V^{2},
$$

which renders the brane mediated mechanism of SSB unviable for phenomenological reasons. This means that the phenomenological hierarchy between the SM typical energy scale of order $\mathrm{TeV}$ and the Planck scale of the gravitational effects of the physics on the brane cannot be accounted by the SSB brane mechanism, since the characteristic scale of the induced dynamics of the scalar field is the Planck scale. It is easy to see that $\langle\Phi\rangle \sim M_{P l(4)}$. Thus, the scalar field becomes a short range field about the brane and therefore strongly localized therein. However, it is the non-minimal coupling that, upon spontaneous symmetry breaking, allows the matter localized on the brane to interact with bulk matter fields with typically gravitational strength.

Moreover, from the expression for the five-dimensional cosmological constant in the case of a vanishing effective cosmological constant, we find that $\Lambda_{(5)} \sim-M_{\phi} V\left(\langle\Phi\rangle^{2}\right)-\sigma^{2} \kappa_{(5)}^{2}$, and consequently that

$$
\sigma^{2} \sim M_{P l(4)}^{2} M_{\phi}\left[-\Lambda_{(5)}-M_{\phi} V\left(\langle\Phi\rangle^{2}\right)\right] .
$$

\subsection{The effective potential as a measure of the thickness of the brane}

In what follows we argue that the mass scale generated by the SSB mechanism sets the range of the fields on the brane and hence the thickness of the brane. In order to understand the role of the mass $M_{\phi}$, we expand the vev of $\Phi$ observed on the brane about the value expected in its absence. Defining

$$
\mu_{\sigma}^{2}=2 \sigma^{2} \xi \kappa_{(5)}^{4}, \quad \lambda_{\sigma}=M_{\phi} \sigma^{2} \xi^{2} \kappa_{(5)}^{6},
$$

then equations (2.30) and (2.31) become

$$
\begin{aligned}
\mu_{e f f}^{2} & \sim \mu_{(5)}^{2}+\mu_{\sigma}^{2}, \\
M_{\phi} \lambda_{e f f} & \sim M_{\phi} \lambda_{(5)}+\lambda_{\sigma} .
\end{aligned}
$$

It follows that

$$
\begin{aligned}
-\langle\Phi\rangle^{2}=\frac{\mu_{e f f}^{2}}{M_{\phi} \lambda_{e f f}} & \sim\left(\mu_{(5)}^{2}+\mu_{\sigma}^{2}\right) \frac{1}{M_{\phi} \lambda_{(5)}}\left(1-\frac{\lambda_{\sigma}}{M_{\phi} \lambda_{(5)}}+O\left[\left(\frac{\lambda_{\sigma}}{M_{\phi} \lambda_{(5)}}\right)^{2}\right]\right) \\
& \sim \frac{\mu_{(5)}^{2}}{M_{\phi} \lambda_{(5)}}-\frac{\mu_{\sigma}^{2}}{M_{\phi} \lambda_{(5)}} \frac{\lambda_{\sigma}}{M_{\phi} \lambda_{(5)}}+\frac{\mu_{\sigma}^{2}}{M_{\phi} \lambda_{(5)}}+O\left[\left(\mu_{\sigma}^{2}\right)^{2}, \lambda_{\sigma}^{2}\right]
\end{aligned}
$$

keeping only the lowest power in the parameters indexed $\sigma$. We can now distinguish between two cases, depending on which $\sigma$-indexed term on the right hand side dominates.

For the case when the second term dominates over the third, then

$$
\frac{\mu_{e f f}^{2}}{M_{\phi} \lambda_{e f f}}>\frac{\mu_{(5)}^{2}}{M_{\phi} \lambda_{(5)}} \Rightarrow\left|\frac{\mu_{e f f}^{2}}{M_{\phi} \lambda_{e f f}}\right|<\left|\frac{\mu_{(5)}^{2}}{M_{\phi} \lambda_{(5)}}\right| .
$$


For an increasingly important contribution of the brane tension, both the minimum and the inflexion point of the effective potential converge to $\langle\Phi\rangle \rightarrow 0$, whereas $V_{\text {eff }} \rightarrow \lambda_{\text {eff }}\left(\Phi^{4} / 4\right)$.

For the case when the third term dominates over the second, then the opposite relation holds and the minimum and the inflexion points become larger with an increasing brane contribution. We observe that the presence of the brane affects the characteristics of the potential and consequently the SSB mechanism and the scales generated thereby. Thus, it is in order to compare $\langle\Phi\rangle$ with the inverse of the thickness of the brane, estimated to be of order the effective mass $\mu_{\text {eff }}$. We find that $M_{\phi} \lambda_{\text {eff }} \sim 1$. From Eq. (2.34), this also implies that $\mu_{\text {eff }}^{2} \sim M_{P l(4)}^{2}$.

The present analysis can be extended to a complex scalar field $\Psi$, coupled to a $U(1)$ gauge, resulting quite similar conclusions [3]. Both the complex scalar field and the $U(1)$ vector field acquire, upon SSB and through a non-minimal coupling of the form $\Psi \bar{\Psi} R$, masses of order the Planck mass as well.

\section{Discussion}

In this contribution we have examined the mechanism of spontaneous symmetry breaking due to a scalar field in the bulk spacetime coupled non-minimally to gravity. We have shown that a non-minimal coupling can be a source of symmetry breaking on the brane but only at very high energies. We derived the conditions which allow for the existence of a non-vanishing bulk scalar field vacuum configuration and demonstrated that the scales of the induced masses are of order the four-dimensional Planck scale, thus failing to accommodate on the brane the typical scales of the Standard Model. We notice, however, that this implies that the bulk scalar fields become very short range about the position of the brane and thus strongly localized therein.

Furthermore, we observe that in the absence of the non-minimal coupling of the bulk scalar fields to gravity, i.e. for $\xi=0$, the effective potential on the brane of a bulk scalar field reduces in both cases to $V_{e f f}=\mu_{(5)}^{2}\left(\phi^{2} / 2\right)+\lambda_{(5)}\left(\phi^{4} / 4\right)+(3 / 4) \sigma^{2} \kappa_{(5)}^{2} d^{2} /[(d+1)(d-1)]$. The realization of a braneworld universe as a codimension-one surface of localized matter contributes a constant term proportional to the brane tension $\sigma$ to the effective potential. The brane tension does not, however, contribute to the mechanism of spontaneous symmetry breaking observed on the brane unless the bulk scalar fields are non-minimally coupled to gravity. This is observed in the dependence on the coupling parameter $\xi$ of the parameters $\mu_{e f f}^{2}$ and $\lambda_{e f f}$. Moreover, the mixing of the discontinuity in the extrinsic curvature with the discontinuity in the normal derivative of the scalar field, as encompassed by the corresponding matching conditions, is also $\xi$-dependent. Such mixing is switched off when $\xi=0$, as already noticed in Ref. [6] and also found in Ref. [5]. These observations seem to suggest that the matter localized on the brane will interact with bulk matter fields through gravity only if a non-minimal coupling exists. We have also argued that the characteristics of the potential, and in particular the vev of the scalar field, can be related to the estimated thickness of the brane, given that the former sets the effective range of the fields. This suggests that the SSB mechanism is closely related to the localization process of fields on the brane.

On the technical side, our approach goes a step further in setting up the framework for studying the brane induced physics which arises from the presence of matter fields in the bulk. The case of a vector field coupled non-minimally to gravity was previously considered and its implications for Lorentz symmetry on the brane studied in Ref. [5]. The case of the scalar field, both real and 
complex, coupled non-minimally to gravity is treated in Ref. [3]. Our approach allows to relate the cosmological constant problem and the scale of gravity to the mechanism of origin of mass, which suggests to relate to the process of localization of bulk fields on the brane by setting the nature of the interaction between brane and bulk fields to be essentially gravitational.

\section{Acknowledgments}

C. C. thanks Fundação para a Ciência e a Tecnologia - FCT (Portuguese Agency) for financial support under the fellowship /BPD/18236/2004. O. B. acknowledges the partial support of the FCT project POCI/FP/63916/2005 and of the European Science Foundation network programme "Quantum Geometry and Quantum Gravity". O. B. also expresses his gratitude to Florian Girelli and Stefano Liberati for the kind hospitality at Trieste. The authors thank Martin Bucher, Mariam Bouhmadi Lopez, Fernando Quevedo and Kyriakos Tamvakis for useful discussions.

\section{A. Geometry and Matter Sources}

In this section we systematize the procedure that we used to derive the equations of motion on a codimension-one brane from a given bulk action.

We begin to parametrize the worldsheet in terms of coordinates $x^{A}=\left(t_{b}, \mathbf{x}_{b}\right)$ intrinsic to the brane [7]. Using the chain rule, we may express the brane tangent and normal unit vectors in terms of the bulk basis as follows:

$$
\begin{aligned}
& \hat{e}_{A}=\frac{\partial}{\partial x^{A}}=X_{A}^{\mu} \frac{\partial}{\partial x^{\mu}}=X_{A}^{\mu} \hat{e}_{\mu} \\
& \hat{e}_{N}=\frac{\partial}{\partial n}=N^{\mu} \frac{\partial}{\partial x^{\mu}}=N^{\mu} \hat{e}_{\mu}
\end{aligned}
$$

with

$$
g_{\mu v} N^{\mu} N^{v}=1, \quad g_{\mu v} N^{\mu} X_{A}^{v}=0
$$

where $\mathbf{g}$ is the bulk metric

$$
\mathbf{g}=g_{\mu \nu} \hat{e}_{\mu} \otimes \hat{e}_{v}=g_{A B} \hat{e}_{A} \otimes \hat{e}_{B}+g_{A N} \hat{e}_{A} \otimes \hat{e}_{N}+g_{N B} \hat{e}_{N} \otimes \hat{e}_{B}+g_{N N} \hat{e}_{N} \otimes \hat{e}_{N}
$$

To obtain the metric induced on the brane we expand the bulk basis vectors in terms of the coordinates intrinsic to the brane and keep the doubly brane tangent components only. It follows that

$$
g_{A B}=X_{A}^{\mu} X_{B}^{v} g_{\mu v}
$$

is the $(3+1)$-dimensional metric induced on the brane by the $(4+1)$-dimensional bulk metric $g_{\mu \nu}$. The induced metric with upper indices is defined by the relation

$$
g_{A B} g^{B C}=\delta_{A}{ }^{C} .
$$

It follows that we can write any bulk tensor field as a linear combination of mutually orthogonal vectors on the brane, $\hat{e}_{A}$, and a vector normal to the brane, $\hat{e}_{N}$. We illustrate the example of a vector 
$B_{\mu}$ and a tensor $T_{\mu \nu}$ bulk fields as follows

$$
\begin{aligned}
& \mathbf{B}=B_{A} \hat{e}_{A}+B_{N} \hat{e}_{N}, \\
& \mathbf{T}=T_{A B} \hat{e}_{A} \otimes \hat{e}_{B}+T_{A N} \hat{e}_{A} \otimes \hat{e}_{N}+T_{N B} \hat{e}_{N} \otimes \hat{e}_{B}+T_{N N} \hat{e}_{N} \otimes \hat{e}_{N} .
\end{aligned}
$$

Derivative operators decompose similarly. We write the derivative operator $\nabla$ as

$$
\nabla=\left(X_{A}^{\mu}+N^{\mu}\right) \nabla_{\mu}=\nabla_{A}+\nabla_{N}
$$

Choosing Fermi normal coordinates for the neighborhood of a point on the brane, all the Christoffel symbols of the metric on the boundary are zero. The partial derivatives, however, do not vanish in general. The continuation of the coordinates off the boundary is given by the Gaussian normal prescription. The non-vanishing connection coefficients are thus

$$
\begin{aligned}
& \nabla_{A} \hat{e}_{B}=-K_{A B} \hat{e}_{N}, \\
& \nabla_{A} \hat{e}_{N}=+K_{A B} \hat{e}_{B}, \\
& \nabla_{N} \hat{e}_{A}=+K_{A B} \hat{e}_{B}, \\
& \nabla_{N} \hat{e}_{N}=0 .
\end{aligned}
$$

For the derivative operator $\nabla \nabla$ we find that

$$
\begin{aligned}
\nabla \nabla & =g^{\mu v} \nabla_{\mu} \nabla_{v} \\
& =g^{A B}\left[\left(X_{A}^{\mu} \nabla_{\mu}\right)\left(X_{B}^{v} \nabla_{v}\right)-X_{A}^{\mu}\left(\nabla_{\mu} X_{B}^{v}\right) \nabla_{v}\right]+g^{N N}\left[\left(N^{\mu} \nabla_{\mu}\right)\left(N^{v} \nabla_{v}\right)-N^{\mu}\left(\nabla_{\mu} N^{v}\right) \nabla_{v}\right] \\
& =g^{A B}\left[\nabla_{A} \nabla_{B}+K_{A B} \nabla_{N}\right]+\nabla_{N} \nabla_{N} .
\end{aligned}
$$

We can now decompose the Riemann tensor, $R_{\mu v \rho \sigma}$, along the tangent and the normal directions to the surface of the brane as follows

$$
\begin{aligned}
& R_{A B C D}=R_{A B C D}^{(i n d)}+K_{A D} K_{B C}-K_{A C} K_{B D} \\
& R_{N B C D}=K_{B C ; D}-K_{B D ; C} \\
& R_{N B N D}=K_{B C} K_{D C}-K_{B D, N}
\end{aligned}
$$

from which we find the decomposition of the Einstein tensor, $G_{\mu \nu}$, obtaining the Gauss-Codacci relations

$$
\begin{aligned}
G_{A B} & =G_{A B}^{(i n d)}+2 K_{A C} K_{B}^{C}-K_{A B} K-K_{A B, N}-\frac{1}{2} g_{A B}\left(-K_{C D} K^{C D}-K^{2}-2 K_{, N}\right), \\
G_{A N} & =K_{A}{ }^{B} ; B-K_{; A}, \\
G_{N N} & =\frac{1}{2}\left(-R^{(i n d)}-K_{C D} K^{D C}+K^{2}\right) .
\end{aligned}
$$

We have now the tools to express the bulk equations of motion derived from the bulk action as a decomposition along the parallel and orthogonal directions to the brane as defined by the Gaussian normal prescription.

Next we define the boundary conditions compatible with the presence of the brane. We regard the brane as shell of thickness $2 \delta$ in the limit $\delta \rightarrow 0$ and separating the bulk into two mirroring regions. Two consequences follow. Derivatives of quantities discontinuous across the brane generate singular distributions on the brane. The integration of these terms in the coordinate normal 
to the brane relates the induced geometry with the localization of the induced stress-energy in the form of matching conditions. Moreover, the $Z_{2}$-symmetry establishes the continuity conditions for the fields across the brane. From the continuity of the quantities on the brane, it follows that the parallel components must be even in $N$ and consequently that the orthogonal components must be odd in $N$, with each additional orthogonal component reverting the parity. [See Ref. [8] for odd fields about the brane.] In particular, for a scalar field $\phi$ and a vector field $\mathbf{B}$ we have that

$$
\begin{aligned}
\phi(-\delta) & =+\phi(+\delta), & & \left(\nabla_{N} \phi\right)(-\delta)=-\left(\nabla_{N} \phi\right)(+\delta) \\
B_{A}(-\delta) & =+B_{A}(+\delta), & & \left(\nabla_{N} B_{A}\right)(-\delta)=-\left(\nabla_{N} B_{A}\right)(+\delta) \\
B_{N}(-\delta) & =-B_{N}(+\delta), & & \left(\nabla_{N} B_{N}\right)(-\delta)=+\left(\nabla_{N} B_{N}\right)(+\delta)
\end{aligned}
$$

Similarly, we have for the metric that $g_{A B}(N=-\delta)=+g_{A B}(N=+\delta)$, which implies that

$$
K_{A B}(N=-\delta)=-K_{A B}(N=+\delta) .
$$

After extracting the singular terms from the projected equations and into the matching condition, we obtain the equations of motion induced on the brane. Since the original equations were derived from a bulk action, the induced ones will be expressed in terms of bulk parameters, which must be rescaled in order to reproduce the observed characteristically four-dimensional parameters. The rescaling parameter is expected to be related to a measure of the thickness of the brane.

\section{References}

[1] G.R. Dvali and M.A. Shifman, Domain walls in strongly coupled theories, Phys. Lett. B396 (1997) 64, Erratum-ibid. B407 (1997) 452.

[2] K. Farakos and P. Pasipoularides, Gravity-induced instability and gauge field localization, Phys. Lett. B621 (2005) 224.

[3] O. Bertolami and C. Carvalho, Spontaneous Symmetry Breaking in the Bulk and the Localization of Fields on the Brane, hep-th/0705.1923; to appear in Phys. Rev. D.

[4] M. Laine, H.B. Meyer, K. Rummukainen and M. Shaposhnikov, Effective gauge theories on domain walls via bulk confinement?, JHEP 0404 (2004) 027.

[5] O. Bertolami and C. Carvalho, Lorentz Symmetry Derived from Lorentz Violation in the Bulk, Phys. Rev. D 74 (2006) 084020 (see also O. Bertolami and C. Carvalho, gr-qc/0612129 for further discussions).

[6] C. Barceló and M. Visser, Moduli fields and brane tension: generalizing the junction conditions, Phys. Rev. D 63 (2001) 02400.

[7] M. Bucher and C. Carvalho, Linearized Israel matching conditions for cosmological perturbations in a moving brane background, Phys. Rev. D 71 (2005) 083511.

[8] T. Gherghetta and A. Pomarol, Bulk fields and supersymmetry in a slice of AdS, Nucl. Phys. B586 (2000) 141. 